Pacific Journal of Mathematics

FUNDAMENTAL DOMAINS FOR THE GENERAL LINEAR 


\title{
FUNDAMENTAL DOMAINS FOR THE GENERAL LINEAR GROUP
}

\section{Douglas Grenier}

\begin{abstract}
Historically the most familiar fundamental domain for $P_{n} / \mathrm{GL}_{n}(\mathrm{Z})$ has been that of Minkowski. This paper develops a new fundamental domain more suited to applications in number theory. It is shown that these domains can be determined explicitly for given $n$ and this is done for $n=3,4,5,6$. A reduction algorithm for an arbitrary element of $P_{n}$ is also determined.
\end{abstract}

1. Introduction. Throughout this paper, let $P_{n}$ denote the space of positive definite, symmetric, real $n \times n$ matrices. The identity matrix will always be denoted by $I$ or $I_{n}$ where necessary to avoid ambiguity. If $G=\mathrm{GL}_{n}(\mathbf{R})$, the general linear group over $\mathbf{R}$, and $K$ is the subgroup of $G$ of orthogonal matrices, $P_{n}$ can be identified with $K \backslash G$ as follows:

$$
\begin{aligned}
K \backslash G & \rightarrow P_{n} \\
K g & \rightarrow{ }^{T} g g
\end{aligned}
$$

where ${ }^{T} g$ denotes the transpose of the matrix $g$. We can define an action of the group $G$ on $P_{n}$ by ${ }^{T} g Y g$ for $g \in G$ and $Y \in P_{n}$. We will use the notation $Y[g]={ }^{T} g Y g$. Now, as $\mathrm{GL}_{n}(\mathbf{Z})$ is a discrete subgroup of $G$, and so acts discontinuously on $P_{n}$, we can define a fundamental domain $P_{n} / \mathrm{GL}_{n}(\mathrm{Z})$. If $\Gamma$ is any discrete subgroup of $G$, then a fundamental domain for $P_{n} / \Gamma$ is a subset of $P_{n}$ satisfying two conditions:

(1) The union of the images under the action of $\Gamma$ covers $P_{n}$, i.e.,

$$
\bigcup_{\gamma \in \Gamma} \gamma\left(P_{n} / \Gamma\right)=P_{n}
$$

(2) If $Y$ and $Y[g], g \in \Gamma$, are both in the fundamental domain, then $Y$ and $Y[g]$ are on the boundary of the fundamental domain or $g=I$. From here on, unless otherwise noted, $\Gamma$ will always be $\mathrm{GL}_{n}(\mathbf{Z})$.

Historically, the standard fundamental domain for $P_{n} / \Gamma$ has been that of Minkowski, [9], here denoted $M_{n} . M_{n}$ is defined as follows:

$$
\begin{array}{r}
M_{n}=\left\{Y \in P_{n} \mid Y[a] \geq y_{i i} \text { if } a \in \mathbf{Z}^{n}, \text { g.c.d. }\left(a_{i}, \ldots, a_{n}\right)=1 ;\right. \\
\left.y_{i, i+1} \geq 0 \text { for } i=1, \ldots, n-1\right\} .
\end{array}
$$


It may not be readily apparent that this indeed satisfies conditions 1 and 2 above, but for the details the reader is referred to Minkowski's original work [9], or to Terras' discussion in [17].

Fundamental domains are by no means unique, however, and while study of $M_{n}$ has led to great progress in the geometry of numbers for example, for certain areas of number theory it may be appropriate to define an alternative fundamental domain. One motivation was to explicitly determine the Maass-Selberg relations for Eisenstein series on $\mathrm{SL}_{3}(Z)$. For $\mathrm{SL}_{2}(Z)$ these enable one to solve the integral that is the parabolic term in the Selberg trace formula. To study the corresponding term in a version of the trace formula for $\mathrm{SL}_{3}(Z)$ it would be necessary to integrate the product of two Eisenstein series on a portion of the fundamental domain. The Minkowski domain does not appear to be well-suited for this. In [18] the problems of Minkowski's fundamental domain for this type of work are discussed in detail. The alternative fundamental domain will be defined in such a way as to avoid these problems. The other major motivation for studying a new fundamental domain was to provide a workable reduction algorithm that could be applied to various number theoretic problems. The fundamental domain defined in this paper will address both of these issues.

The approach is a generalization of the classical "highest point method" used to determine the well-known fundamental domain for the Poincaré upper half-plane. Recall that if $H$ is the upper half-plane, i.e., the subset of complex numbers $z=x+i y$ with $y>0$, a fundamental domain is given by $D=\left\{z=x+i y \in H|| x \mid \leq 1 / 2, x^{2}+y^{2} \geq 1\right\}$. This new fundamental domain bears more than a slight resemblance to the fundamental domain in the case of Siegel upper half-plane, $H_{n}$, which is yet another generalization of the highest point method. Define

$$
H_{n}=\left\{Z=X+i Y \mid X, Y \in \mathbf{R}^{n \times n}, X \text { symmetric, } Y \in P_{n}\right\} .
$$

The symplectic group $\operatorname{Sp}_{n}(Z)$ defined by

$$
\operatorname{Sp}_{n}(\mathbf{Z})=\left\{M \in \mathbf{Z}^{2 n \times 2 n} \mid J[M]=J \text { where } J=\left(\begin{array}{cc}
0 & I_{n} \\
-I_{n} & 0
\end{array}\right)\right\}
$$

acts on the space $H_{n}$ by $Z \rightarrow(A Z+B)(C Z+D)^{-1}$ where $M \in \operatorname{Sp}_{n}(\mathbf{Z})$ is written as $M=\left(\begin{array}{ll}A & B \\ C & D\end{array}\right)$ and $A, B, C, D$ are $n \times n$ block integer matrices. Note that for $n=1, \operatorname{Sp}_{n}(Z)=\mathrm{SL}_{2}(Z)$, and this generalization reduces to the classical case. Under this action a fundamental domain for 
$H_{n} / \mathrm{Sp}_{n}(\mathbf{Z})$ is given by the subset of $Z \in H_{n}$ such that

(1) $|\operatorname{det}(C Z+D)| \geq 1$ for all $C, D$ with $\left(\begin{array}{ll}A & B \\ C & D\end{array}\right) \in \operatorname{Sp}_{n}(\mathbf{Z})$

(2) $Y \in M_{n}$

(3) $X=\left(x_{i j}\right),\left|x_{i j}\right| \leq \frac{1}{2}$.

E. Gottschling has shown, [3], that for the case $n=2$ the necessary $C$ and $D$ in condition (1) are $C=I$ and $D$ is one of 15 choices, explicitly determined, all with entries $0, \pm 1$, or $C$ is a rank 1 matrix in which case if $Z=\left(\begin{array}{cc}z_{1} & z_{12} \\ z_{12} & z_{2}\end{array}\right)$ then $\left|z_{1}\right|,\left|z_{2}\right| \geq 1$ and $\left|z_{1}-2 z_{12}+z_{2} \pm 1\right| \geq 1$. We will achieve analogous results using somewhat similar reasoning for the fundamental domain $P_{n} / \Gamma$ for $n=3,4,5,6$. For more details on the Siegel modular group, in particular the proof that the set defined above is actually a fundamental domain for $H_{n} / \mathrm{Sp}_{n}(\mathbf{Z})$ see [8].

All of the aforementioned groups and spaces are related. For example, if $S P_{n}$ denotes the subspace of $P_{n}$ of those matrices with determinant 1 , and $\mathrm{SL}_{n}(\mathbf{Z})$ is the special linear group of integral matrices with determinant 1 , the case $n=2$ is once again the classical situation, for $S P_{2}$ and $H$ can be identified by:

$$
\begin{gathered}
H \rightarrow S P_{2} \\
x+i y=z \rightarrow\left(\begin{array}{cc}
y^{-1} & 0 \\
0 & y
\end{array}\right)\left[\begin{array}{ll}
1 & x \\
0 & 1
\end{array}\right] .
\end{gathered}
$$

More generally, $H_{n}$ can be embedded in $S P_{n}$ by

$$
X+i Y=Z \rightarrow\left(\begin{array}{cc}
Y^{-1} & 0 \\
0 & Y
\end{array}\right)\left[\begin{array}{cc}
I & X \\
0 & I
\end{array}\right] .
$$

So perhaps study of our fundmental domain for $P_{n}$ may lead to an increased understanding in all these areas, through these relations. For the moment, however, the applications seem to be in the theory of automorphic forms on $\mathrm{GL}_{n}(\mathbf{Z})$.

2. Partial Iwasawa co-ordinates. Before we can define the new fundamental domain, we must first describe the system of co-ordinates. For $Y \in P_{n}$ what is sometimes called the Iwasawa decomposition is given by

$$
Y=\left(\begin{array}{lll}
d_{1} & & 0 \\
& \ddots & \\
0 & & d_{n}
\end{array}\right)\left[\begin{array}{ccc}
1 & & x_{i j} \\
& \ddots & \\
0 & & 1
\end{array}\right]
$$

with $d_{i}>0$ and $x_{i j} \in \mathbf{R}$. This is the co-ordinate system Minkowski employed to find his fundamental domain. For the new domain we will want to look at a generalization of Iwasawa co-ordinates. These 
can be expressed as:

$$
Y=\left(\begin{array}{cc}
V & 0 \\
0 & W
\end{array}\right)\left[\begin{array}{cc}
I_{j} & X \\
0 & I_{k}
\end{array}\right]
$$

where $V \in P_{j}, W \in P_{k}, X \in \mathbf{R}^{j \times k}$ and of course $j+k=n$. Clearly, there are at least $n-1$ different types of partial Iwasawa co-ordinates. Partial Iwasawa co-ordinates are actually nothing more than a generalization of completing the square. For our purposes, it becomes clear that the best choice is:

$$
Y=\left(\begin{array}{cc}
v & 0 \\
0 & W
\end{array}\right)\left[\begin{array}{cc}
1 & T_{X} \\
0 & I_{n-1}
\end{array}\right]
$$

where $v>0, W \in P_{n-1}, x \in \mathbf{R}^{n-1}$, i.e., ${ }^{T} x=\left(x_{1}, \ldots, x_{n-1}\right), x_{j} \in \mathbf{R}$. As will be seen later, with this choice of co-ordinates we can avoid having to use a determinant, as $v$ is just a scalar.

We are also going to consider what the group action of $\mathrm{GL}_{n}(\mathbf{Z})$ on $P_{n}$ looks like in terms of these co-ordinates. First, for an element $g \in \Gamma$, write $g=\left(\begin{array}{ll}a^{T} b \\ c & D\end{array}\right)$ for $a \in \mathbf{Z},{ }^{T} b, c \in \mathbf{Z}^{n-1}$ and $D \in \mathbf{Z}^{n-1 \times n-1}$. This corresponds to the specific type of partial Iwasawa co-ordinates being used. Then

$$
\begin{aligned}
Y[g] & =\left(\begin{array}{cc}
v & 0 \\
0 & W
\end{array}\right)\left[\begin{array}{cc}
1 & { }^{T} x \\
0 & I
\end{array}\right]\left[\begin{array}{cc}
a & { }^{T} b \\
c & D
\end{array}\right] \\
& =\left(\begin{array}{cc}
v & v^{T} x \\
v x & v\left[{ }^{T} x\right]+W
\end{array}\right)\left[\begin{array}{ll}
a & { }^{T} b \\
c & D
\end{array}\right] \\
& =\left(\begin{array}{cc}
a & T_{c} \\
b & { }^{T} D
\end{array}\right)\left(\begin{array}{cc}
v & v^{T} x \\
v x & v\left[{ }^{T} x\right]+W
\end{array}\right)\left(\begin{array}{cc}
a & { }^{T} b \\
r c & D
\end{array}\right) \\
& =\left(\begin{array}{cc}
v\left[a+{ }^{T} x c\right]+W[c] & \left(a+{ }^{T} x c\right) v\left({ }^{T} b+{ }^{T} x D\right)+{ }^{T} c W D \\
\left(b+{ }^{T} D x\right) v\left(a+{ }^{T} x c\right)+{ }^{T} D W c & v\left[{ }^{T} b+{ }^{T} x D\right]+W[D]
\end{array}\right) .
\end{aligned}
$$

The square bracket notation applies to a column vector as well as to a matrix, that is, for $c \in \mathbf{Z}^{n-1}, W[c]={ }^{T} c W c$ which of course is a scalar. Then, since $a+{ }^{T} X c$ is itself scalar, $v\left[a+{ }^{T} X c\right]=$ $v\left(a+T_{x c}\right)^{2}$. Also, note that the term in the upper left corner must also be scalar. What we mean by $v\left[{ }^{T} x\right],{ }^{T} X$ now being a row of length $n-1$, is $v\left[{ }^{T} x\right]=v X$ where $X$ is the symmetric matrix $\left(x_{i} x_{j}\right)$. The scalar term $v\left[a+{ }^{T} x c\right]+W[c]$ will figure most prominently in the following generalization of the highest point method.

3. The fundamental domain. We are now nearly ready to define the fundamental domain. The motivation for this definition came from looking at $Y[g]$ as above and observing that the upper left corner of $Y[g]$ is $v\left[a+{ }^{T} x c\right]+W[c]$. If we think of the "height" of $Y$ as $v^{-1}$ then we want $Y$ in the fundamental domain to have height greater than that 
of any $Y[g]$. In other words, for $Y$ in the fundamental domain we want $v \leq v\left[a+{ }^{T} x c\right]+W[c]$ for any $a$ and $c$ forming the first column of a matrix in $\Gamma$. The complete definition of the fundamental domain is then as follows.

Definition. For $n \geq 2$, let $F_{n}$ be the set of those $Y \in P_{n}$ satisfying:

(1) $v \leq v\left[a+{ }^{T} x c\right]+W[c]$ where $a \in \mathbf{Z}, c \in \mathbf{Z}^{n-1}$ and $g=\left(\begin{array}{ll}a & { }^{T} b \\ c & D\end{array}\right) \in \Gamma$

(2) $W \in F_{n-1}$

(3) $0 \leq x_{1} \leq \frac{1}{2} ;\left|x_{i}\right| \leq \frac{1}{2}$ for $i=2, \ldots, n-1$.

It will be seen that $F_{n}$ is a fundamental domain for $P_{n} / \mathrm{GL}_{n}(\mathbf{Z})$.

Note the similarity between the definition of $F_{n}$ and the definition of the fundamental domain for the Siegel modular group. Here the height is given by $v^{-1}$; in the Siegel case for $Z \in H_{n}, Z=X+i Y$, the height of $Z$ is given by $\operatorname{det}(Y)$. The first condition arises from maximizing the height. Then the second condition is obtained by looking at $g \in \Gamma$ of the form $g=\left(\begin{array}{cc} \pm 1 & 0 \\ 0 & D\end{array}\right)$ where $g \in \mathrm{GL}_{n-1}(\mathbf{Z})$. This basically sets up an induction; it is therefore, no accident that most of the proofs of statements about the fundamental domain rely on some sort of induction argument. The third condition comes from looking at the "translation-type" matrices, that is, $g \in \Gamma$ of the form $g=\left(\begin{array}{cc}1^{T} b \\ 0 & I\end{array}\right)$. These comments will be examined in more depth in the proof of the theorem in the following section.

We have defined $F_{n}$ above in an inductive manner, that is, it is built up using $F_{n-1}$. In beginning the induction, $F_{1}$ will just be the positive real numbers. However, it is possible to start at $n=2$ using the following proposition.

Proposition 1.

$$
\begin{aligned}
F_{2}=M_{2}=\left\{Y \in P_{n} \mid\right. & 0 \leq 2 y_{12} \leq y_{1} \leq y_{2} \\
& \text { if } \left.y_{12}=0 \text { then } 0<y_{1} \leq y_{2} ; Y=\left(\begin{array}{cc}
y_{1} & y_{12} \\
y_{12} & y_{2}
\end{array}\right)\right\} .
\end{aligned}
$$

Proof. This can easily be seen by examining the first condition in the definition. Since $n=2, Y \in P_{n}$ is expressed as

$$
Y=\left(\begin{array}{cc}
v & 0 \\
0 & w
\end{array}\right)\left[\begin{array}{cc}
1 & x \\
0 & 1
\end{array}\right], \quad v, w>0, \quad x \in \mathbf{R}
$$

Then condition (1) says that $v \leq v(a+x c)^{2}+c^{2} w$ for all $a, c$ such that $g=\left(\begin{array}{ll}a & b \\ c & d\end{array}\right) \in \mathrm{GL}_{2}(\mathbf{Z})$. Choosing $a=0, c=1$ we see that $v \leq v x^{2}+w$ or $w \geq v\left(1-x^{2}\right)$. But condition (3) tells us that $0 \leq x \leq \frac{1}{2}$ so $w \geq \frac{3}{4} v$. It is then apparent that the only $a$ and $c$ we need consider are $a=0, c=1$ 
(compare this with the fundamental domain for $H$, the upper halfplane). By this we mean that $v \leq v(a+x c)^{2}+c^{2} w$ for $a=0, c=1$ implies this for all other choices of $a$ and $c$. Thus we have

$$
F_{2}=\left\{Y \in P_{n} \mid Y=\left(\begin{array}{cc}
v & 0 \\
0 & w
\end{array}\right)\left[\begin{array}{cc}
1 & x \\
0 & 1
\end{array}\right], v \leq v x^{2}+w, 0 \leq x \leq \frac{1}{2}\right\} .
$$

But

$$
\left(\begin{array}{cc}
v & 0 \\
0 & w
\end{array}\right)\left[\begin{array}{cc}
1 & x \\
0 & 1
\end{array}\right]=\left(\begin{array}{cc}
v & v x \\
v x & v x^{2}+w
\end{array}\right)=\left(\begin{array}{cc}
y_{1} & y_{12} \\
y_{12} & y_{2}
\end{array}\right)
$$

and so we have $0 \leq 2 y_{12} \leq y_{1} \leq y_{2}$.

This type of argument will resurface in $\S 6$. Even without working through the proof above, we should have expected that $F_{2}=M_{2}$ is reasonable, for the partial Iwasawa decomposition we are using reduces in the case $n=2$ to the full Iwasawa decomposition which is what Minkowski used for his fundamental domain. Now we have explicit inequalities for $F_{2}$; in $\S 6$ we will determine explicit inequalities for some higher $n$. However, first we must prove that the set $F_{n}$ defined above is in fact a fundamental domain.

4. The highest point method. The following Lemma will be needed in the proof of the Theorem in this section and in later sections as well.

LEMMA 1. If $Y \in F_{n}, Y=\left(\begin{array}{cc}v & 0 \\ 0 & W\end{array}\right)\left[\begin{array}{cc}1^{T} & x \\ 0 & 1\end{array}\right]$ and $w_{i}$ is the ith diagonal entry in $W$, then $w_{i} \geq \frac{3}{4} v$.

Proof. $Y \in F_{n} \Rightarrow v \leq v\left[a+{ }^{T} x c\right]+W[c]$ for all $g=\left(\begin{array}{cc}a^{T} b \\ c & D\end{array}\right) \in \Gamma$. Let $a=0, c=e_{i}$, the standard unit vector in $\mathbf{R}^{n-1}$. Then a $g \in \Gamma$ with $a$ and $c$ forming the first column can be obtained by switching the first and $i$ th rows in the identity matrix. Then $v\left[a+T_{x c}\right]=v x_{i}^{2}$. Since $W\left[e_{i}\right]=w_{i}$, we have $v \leq v x_{i}^{2}+w_{i}$, so $w_{i} \geq v\left(1-x_{i}^{2}\right)$. But $x_{i}^{2} \leq \frac{1}{4}$ so $w_{i} \geq \frac{3}{4} v$.

THEOREM 1. (1) $F_{n}$ as defined above is a fundamental domain for $P_{n} / \Gamma$.

(2) $F_{n}$ has a finite number of boundary inequalities and therefore is bounded by a finite number of hypersurfaces. The minimal set of inequalities can be explicitly obtained for given $n$.

(3) The subset of $Y \in F_{n}$ with $\operatorname{det}(Y) \leq 1$ has finite Euclidean volume given by

$$
\frac{2}{n+1} \prod_{j=2}^{n} \Lambda\left(\frac{j}{2}\right) \quad \text { where } \Lambda(s)=\pi^{-s} \Gamma(s) \zeta(2 s) .
$$


For $F_{n}$ to be useful for any applications we need (2). The proof of (3) depends only on the fact that $F_{n}$ is a fundamental domain, and so is the same proof as for $M_{n}$ given by Siegel [13], or Terras [17], for example. The statement is included here solely for the sake of completeness.

Proof. (1) To prove that $F_{n}$ is a fundamental domain we need to show two things: first, that $\bigcup_{g \in \Gamma} g F_{n}$ covers $P_{n}$, and second, that for $Y$ and $Y[g]$ both in $F_{n}, Y$ and $Y[g]$ must be on the boundary of $F_{n}$, or $g= \pm I$. We have already proved (1) and (2) for $n=2$ in Proposition 1 , so assume that $F_{n-1}$ is a fundamental domain. Then choose $Y \in P_{n}$ :

$$
Y=\left(\begin{array}{cc}
v & 0 \\
0 & W
\end{array}\right)\left[\begin{array}{cc}
1 & T_{X} \\
0 & I
\end{array}\right]
$$

For $Y[g], g=\left(\begin{array}{cc}a^{T} b \\ c & D\end{array}\right)$, let $v^{*}=v\left[a+T^{T} X c\right]+W[c]$, so $v^{*}$ is the upper left corner of $Y[g]$. We must show that there are only finitely many $a$ and $c$ forming the first column of a matrix in $\mathrm{GL}_{n}(\mathbf{Z})$ such that given any positive real number $m, v^{*} \leq m$. If $\lambda$ is the smallest eigenvalue of $W$, then $W[c] \geq \lambda I[c]$ since $W-\lambda I$ will also be positive. Then $W[c] \geq \lambda\left(c_{1}^{2}+\cdots+c_{n-1}^{2}\right)$ and there can be only finitely many $c$ such that $W[c] \leq m$. Once all $c$ have been determined, we must have $v\left(a+T_{x c}\right)^{2} \leq m-W[c]$. For each $c$ there will be only finitely many $a$ satifying this inequality. Since there are only finitely many $a$ and $c$ so that $v^{*} \leq m$ we can choose $g \in \Gamma$ as to make $v^{*}$ minimal.

Once the minimal $v^{*}$ has been produced with

$$
Y[g]=\left(\begin{array}{cc}
v^{*} & 0 \\
0 & W^{*}
\end{array}\right)\left[\begin{array}{cc}
1 & T x^{*} \\
0 & I
\end{array}\right]
$$

we can find $D^{*} \in \mathrm{GL}_{n-1}(\mathbf{Z})$ such that $W^{*}\left[D^{*}\right] \in F_{n-1}$ by the induction hypothesis. Then

$$
g^{*}=\left(\begin{array}{cc}
1 & 0 \\
0 & D^{*}
\end{array}\right) \in \Gamma
$$

Let $g_{1}=g g^{*}$. Then

$$
Y\left[g_{1}\right]=\left(\begin{array}{cc}
v^{*} & 0 \\
0 & W^{*}\left[D^{*}\right]
\end{array}\right)\left[\begin{array}{cc}
1 & T^{*} D^{*} \\
0 & I
\end{array}\right] .
$$

Write ${ }^{T} x^{*} D^{*}=\left(\alpha_{1}, \ldots, \alpha_{n-1}\right)$ and choose $b^{*}$ where ${ }^{T} b^{*}=\left(\beta_{1}, \ldots\right.$, $\beta_{n-1}$ ) so that $\left|\alpha_{j}-\beta_{j}\right| \leq \frac{1}{2}$ for $j=1, \ldots, n-1$. Finally, if $-\frac{1}{2} \leq$ $\alpha_{1}-\beta_{1}<0$, let

$$
g_{2}=g_{1}\left(\begin{array}{cc}
1 & T b^{*} \\
0 & I
\end{array}\right)\left(\begin{array}{cc}
-1 & 0 \\
0 & I
\end{array}\right)
$$


and if $0 \leq \alpha_{1}-\beta_{1} \leq \frac{1}{2}$, let

$$
g_{2}=g_{1}\left(\begin{array}{cc}
1 & T b^{*} \\
0 & I
\end{array}\right)
$$

Then, in either case,

$$
Y\left[g_{2}\right]=\left(\begin{array}{cc}
v_{2} & 0 \\
0 & W_{2}
\end{array}\right)\left[\begin{array}{cc}
1 & T_{u} \\
0 & I
\end{array}\right]
$$

where $v_{2}=v^{*}, W_{2}=W^{*}\left[D^{*}\right]$, and $u= \pm\left(x^{*} D^{*}-b^{*}\right)$, the sign determined as to make $\alpha_{1}-\beta_{1}$ nonnegative. Clearly, conditions (2) and (3) in the definition of $F_{n}$ hold for $Y\left[g_{2}\right]$, so it remains only to show that $v_{2} \leq v_{2}\left[r+T_{u t]}+W_{2}[t] \forall r, t\right.$ with $\left(\begin{array}{cc}r^{T} & U \\ t & U\end{array}\right) \in \Gamma$. If we write $g_{2}\left(\begin{array}{cc}r & T_{S} \\ t & U\end{array}\right)=\left(\begin{array}{cc}r_{1} & T_{S_{1}} \\ t_{1} & U_{1}\end{array}\right)$, then

$$
v_{2}\left[r+T_{u t}\right]+W_{2}[t]=v\left[r_{1}+T_{x t_{1}}\right]+W\left[t_{1}\right]
$$

and

$$
v_{2}=v^{*} \leq v\left[r_{1}+{ }^{T} x t_{1}\right]+W\left[t_{1}\right]=v_{2}\left[r+T^{T} u t\right]+W_{2}[t]
$$

because of how $v^{*}$ was chosen. Thus we have shown that any element of $P_{n}$ can be written as $Y[g], Y \in F_{n}, g \in \Gamma$.

To prove the second condition of being a fundamental domain, again use induction. It has been shown that this condition holds for $n=2$ in Proposition 1, so assume this condition holds for $F_{n-1}$. Now suppose $Y$ and $Y[g]$ are both in $F_{n}$.

$$
Y \in F_{n} \Rightarrow v \leq v\left[a+{ }^{T} x c\right]+W[c] \quad \forall a, c \text { with }\left(\begin{array}{cc}
a & T b \\
c & D
\end{array}\right) \in \Gamma .
$$

By taking the inverse:

$$
Y[g] \in F_{n} \Rightarrow v\left[a+{ }^{T} x c\right]+W[c] \leq v \quad \text { if } g=\left(\begin{array}{cc}
a & T_{b} \\
c & D
\end{array}\right) .
$$

That is, if $Y[g]$ is in the fundamental domain then the upper left corner of $Y[g]$ is less than or equal to the upper left corner of $Y[g][h]$ for any $h \in \Gamma$, including $g^{-1}$, and $Y[g]\left[g^{-1}\right]=Y$. Anyway, the two inequalities above imply that $v=v\left[a+{ }^{T} X c\right]+W[c]$. If $c \neq 0$, then this shows that $Y$ and $Y[g]$ are on the boundary of $F_{n}$, since the inequalities $v \leq v\left[a+T_{X c}\right]+W[c]$ define part of the boundary. If $c=0$, this reduces to $v=v[a]=a^{2} v$ and so $a= \pm 1$.

Then $g=\left(\begin{array}{cc} \pm 1^{T} b \\ 0 & D\end{array}\right)$ and $\operatorname{det}(g)= \pm 1 \Rightarrow \operatorname{det}(D)= \pm 1 \Rightarrow D \in$ $\mathrm{GL}_{n-1}(\mathbf{Z})$

$$
Y[g]=\left(\begin{array}{cc}
v & 0 \\
0 & W[D]
\end{array}\right)\left[\begin{array}{cc}
1 & \pm{ }^{T} x D+{ }^{T} b \\
0 & I
\end{array}\right]
$$


and so $Y, Y[g] \in F_{n} \Rightarrow W, W[D] \in F_{n-1}$ and by the induction hypothesis, either $W$ and $W[D]$ are on the boundary of $F_{n-1}$ or $D= \pm I$. If $W$ and $W[D]$ are on the boundary of $F_{n-1}$, then so are $Y$ and $Y[g]$ on the boundary $F_{n}$, by definition of $F_{n}$. The other case is $D= \pm I$. Then $g=\left(\begin{array}{cc} \pm 1^{T} b \\ 0 & \pm I\end{array}\right)$ and

$$
\begin{aligned}
Y[g]=\left(\begin{array}{cc}
v & 0 \\
0 & W
\end{array}\right)\left[\begin{array}{cc}
1 & \pm{ }^{T} x \pm T^{T} b \\
0 & I
\end{array}\right] \\
Y, Y[g] \in F_{n} \Rightarrow x, \pm x \pm b \in\left[\frac{-1}{2}, \frac{1}{2}\right]^{n-1} \\
\quad \text { with } x_{1}, \pm x_{1} \pm b_{1} \in\left[0, \frac{1}{2}\right]
\end{aligned}
$$

But $b \in \mathbf{Z}^{n-1} \Rightarrow$ either $x_{i}= \pm \frac{1}{2}, b_{i}= \pm 1, i=2, \ldots, n-1 ; x_{1}=\frac{1}{2}, b_{1}=$ 1 or $x_{i} \neq \pm \frac{1}{2}, b_{i}=0$.

So, either $Y$ and $Y[g]$ are on the boundary of $F_{n}$ determined by the $x$ co-ordinates, or all the $b_{i}=0$, in which case $g=\left(\begin{array}{cc} \pm 1 & 0 \\ 0 & \pm 1\end{array}\right)$. If $g=\left(\begin{array}{cc}-1 & 0 \\ 0 & I\end{array}\right)$, then $Y[g]=\left(\begin{array}{cc}v & 0 \\ 0 & W\end{array}\right)\left[\begin{array}{cc}1 & -{ }^{T} x \\ 0 & 1\end{array}\right]$ and both $\pm x_{1} \in\left[0, \frac{1}{2}\right]$, so $x_{1}=0$, and $Y$ and $Y[g]$ are on the boundary. Replacing $g$ with $-g$ yields the same results. Finally, the only possibilities left are $g= \pm I$. Thus, we have seen that either $Y$ and $Y[g]$ are on the boundary of $F_{n}$, or $g= \pm I$.

(2) Again the proof is by induction. Proposition 1 gives the proof for ${ }^{\prime} n=2$. Now assume that there are finitely many boundary inequalities for $F_{n-1}$. The boundary inequalities in condition (3) in the definition of $F_{n}$ are clearly finite in number, and by the induction hypothesis so are those for condition (2). It remains to examine condition (1), $v \leq v\left[a+{ }^{T} x c\right]+W[c]$ for all $a, c$ forming the first column of a matrix $g \in \Gamma$. Now $W \in F_{n-1}$, so write

$$
W=\left(\begin{array}{cc}
v^{\prime} & 0 \\
0 & W^{\prime}
\end{array}\right)\left[\begin{array}{cc}
1 & T_{X^{\prime}} \\
0 & I_{n-2}
\end{array}\right], \quad v^{\prime}>0, W^{\prime} \in F_{n-2}, \quad x^{\prime} \in\left[-\frac{1}{2}, \frac{1}{2}\right]^{n-2} .
$$

By the induction hypothesis the inequalities $v^{\prime} \leq v^{\prime}\left[a^{\prime}+{ }^{T} x^{\prime} c^{\prime}\right]+W^{\prime}\left[c^{\prime}\right]$ are finite in number. But $W[c]=v^{\prime}\left[c_{1}+{ }^{T} x c^{\prime}\right]+W^{\prime}\left[c^{\prime}\right]$ where ${ }^{T} c^{\prime}=$ $\left(c_{2}, \ldots, c_{n-1}\right)$. We now have a finite number of vectors $c$, and together with the bounds on the $x_{i}$, a finite number of $a$ will be forced as well.

To explicitly determine the necessary and sufficient type (1) inequalities, expand $v\left[a+{ }^{T} x c\right]+W[c]$ as follows:

$$
\begin{aligned}
v\left[a+{ }^{T} x c\right]+W[c]= & v\left(a+{ }^{T} x c\right)^{2}+v^{\prime}\left(c_{1}+{ }^{T} x^{\prime} c^{\prime}\right)^{2} \\
& +\cdots+v^{(n-2)}\left(c_{n-2}+x^{(n-2)} c_{n-1}\right)^{2}+v^{(n-1)} c_{n-1}^{2}
\end{aligned}
$$


where $x^{(n-2)}$ is a scalar, and where

$$
\begin{aligned}
Y & =\left(\begin{array}{cc}
v & 0 \\
0 & W
\end{array}\right)\left[\begin{array}{cc}
1 & T_{X} \\
0 & I_{n-1}
\end{array}\right], \\
W & =\left(\begin{array}{cc}
v^{\prime} & 0 \\
0 & W^{\prime}
\end{array}\right)\left[\begin{array}{cc}
q & T_{X^{\prime}} \\
0 & I_{n-2}
\end{array}\right]
\end{aligned}
$$

and so on. Then we arrive at $Y=D[T]$ where $D$ is a diagonal matrix with $v, v^{\prime}, \ldots, v^{(n-1)}$ the diagonal elements and $T$ is an upper triangular matrix with 1 at each diagonal element and with rows consisting of the ${ }^{T} X^{(j)}$ beginning with ${ }^{T} X$ at the top and working our way down in sequence. Now, by repeated application of Lemma 1:

$$
v^{(k)} \geq\left(\frac{3}{4}\right)^{k} v
$$

and thus we arrive at:

$$
\begin{aligned}
v\left[a+{ }^{T} X c\right] & +W[c] \\
\leq v[ & \left(a+{ }^{T} x c\right)^{2}+\frac{3}{4}\left(c_{1}+{ }^{T} x^{\prime} c^{\prime}\right)^{2} \\
& \left.+\cdots+\left(\frac{3}{4}\right)^{n-2}\left(c_{n-2}+x^{(n-2)} c_{n-1}\right)^{2}+\left(\frac{3}{4}\right)^{n-1} c_{n-1}^{2}\right] .
\end{aligned}
$$

If for given $a$ and $c, v\left[a+{ }^{T} x c\right]+W[c] \geq v$ for all $Y \in F_{n}$, the condition $v \leq v\left[a+{ }^{T} x c\right]+W[c]$ for this $a$ and $c$ is superfluous. Thus, we can throw out all $a, c$ that make $\left(a+T_{x c}\right)^{2}+\cdots+\left(\frac{3}{4}\right)^{n-1} c_{n-1}^{2} \geq 1$ for all possible $x, x^{\prime}, \ldots, x^{(n-2)}$. The remaining $a$ and $c$ are necessary and sufficient in condition (1) of the definition of $F_{n}$.

(3) Again, for the proof of part 3 see [17].

Finally, the proof of the Theorem has been completed, so we now know that $F_{n}$ actually is a fundamental domain for $P_{n} / \mathrm{GL}_{n}(\mathbf{Z})$.

5. Geometry on the fundamental domain. Recall that one of the motivations for developing a new fundamental domain as an alternative to Minkowski's fundamental domain, was that Minkowski's fundamental domain proved to not have quite the right "shape" for work on generalizing the Selberg trace formula. In this section we will want to discuss the "shape" of $F_{n}$, in particular to develop a notion of the "cusps" in $F_{n}$, and to show that as a point approaches a cusp, the fundamental domain will have a shape more adaptable to generalizing the trace formula. We will also consider some of the structure of the fundamental domain, especially how it can be built up inductively. Included will be various inequalities relating the entries of $Y \in F_{n}$. 
Of great benefit in this section, as well as quite often in later sections, will be the following Lemma, which can be proved using only elementary linear algebra.

LEMMA 2. There exists

$$
g=\left(\begin{array}{cc}
a & T b \\
c & D
\end{array}\right), \quad g \in \Gamma
$$

if and only if g.c.d. $\left(a, c_{1}, c_{2}, \ldots, c_{n-1}\right)=1$.

Proof. One direction is trivial: if g.c.d. $\left(a, c_{1}, c_{2}, \ldots, c_{n-1}\right)=m>$ 1 , then $m \mid \operatorname{det}(g)$. Now suppose g.c.d. $\left(a, c_{1}, \ldots, c_{n-1}\right)=1$. If we consider the set of matrices obtained from elementary row operations on the identity, and multiply the column vector on the left by these matrices, we can manipulate the vector until we obtain $m e_{1}$ where $m>0$ and $e_{1}$ is the first standard unit vector. Then, if $M$ is the product of the elementary matrices used, $M \in \Gamma$. We now have

$$
M\left(\begin{array}{l}
a \\
c
\end{array}\right)=m e_{1}, \quad\left(\begin{array}{l}
a \\
c
\end{array}\right)=m M^{-1} e_{1} .
$$

Then g.c.d. $\left(a, c_{1}, \ldots, c_{n-1}\right)=1 \Rightarrow m=1$ and $\left(\begin{array}{l}a \\ c\end{array}\right)=M^{-1} e_{1}$ which is the first column of $M^{-1} \in \Gamma$.

The following will also be useful in future calculations.

Lemma 3. Let $Y=\left(y_{i j}\right)$ and for convenience $y_{i}=y_{i l}$. If $Y \in F_{n}$, then

(1) $y_{1} \leq y_{i}$ for $i=1, \ldots, n$

(2) $\left|2 y_{i j}\right| \leq y_{i}, y_{j}$.

Proof. With $Y$ as in (4.1): $Y \in F_{n} \Rightarrow v \leq v\left[a+{ }^{T} x c\right]+W[c]$ for all $a, c$ with g.c.d. $\left(a, c_{1}, \ldots, c_{n-1}\right)=1$. Let $a=0, c=e_{i}, i=1, \ldots, n-1$. Then $v \leq v x_{i}^{2}+w_{i}=y_{i+1}$. Note that $v=y_{1}$. This proves (1).

To prove (2), let $a=0, c=e_{i}-e_{j}$. Then

$$
\begin{aligned}
v[a+ & \left.{ }^{T} x c\right]+W[c]=v\left(x_{i}-x_{j}\right)^{2}+w_{i}-2 w_{i j}+w_{j} \\
& =v x_{i}^{2}+w_{i}-2\left(v x_{i} x_{j}+w_{i j}\right)+v x_{j}^{2}+w_{j} \\
& =y_{i+1}-2 y_{i+1}+1+y_{j+1}
\end{aligned}
$$

and so we have

$$
y_{1}=v \leq y_{i+1}-2 y_{i+1 j+1}+y_{j+1}, \quad 2 y_{i+1}{ }_{j+1} \leq y_{i+1}+y_{j+1}-y_{1} .
$$


Then from (1): $2 y_{i+1} j+1 \leq y_{i+1}, y_{j+1}$. Now let $a=0$ and $c=e_{i}+e_{j}$. Similarly we get

$$
\begin{aligned}
& y_{1} \leq y_{i+1}+2 y_{i+1} j_{+1}+y_{j+1} \\
& 2 y_{i+1} j+1 \geq y_{1}-y_{i+1}-y_{j+1} \\
& 2 y_{i+1} j \geq-y_{i+1},-y_{j+1}
\end{aligned}
$$

and finally $\left|2 y_{i j}\right| \leq y_{i}, y_{j}$.

Now we proceed to obtain some information about determinants of $Y \in F_{n}$. We will use the notation $|Y|$ for the determinant of $Y$. For $Y \in M_{n}$, it is known that $|Y| \leq y_{1} \cdots y_{n} \leq c_{n}|Y|$ for some constant $c_{n}$ depending only on $n$. The first inequality actually holds for $Y \in$ $P_{n}$. For more details on the constant $c_{n}$ see Terras [17]. For the fundamental domain $F_{n}$ the analogous statement is much simpler to state and prove as evidenced by the following Theorem.

THEOREM 2. For $Y \in F_{n}$,

$$
|Y| \leq y_{1} \cdots y_{n} \leq\left(\frac{4}{3}\right)^{n(n-1) / 2}|Y|
$$

Then we have

$$
|Y| \geq\left(\frac{4}{3}\right)^{n(n-1) / 2} y_{1}^{n}
$$

Proof. As already mentioned, the inequality $|Y| \leq y_{1} \cdots y_{n}$ is true for all $Y \in P_{n}$. To see this, write $Y \in P_{n}$ in the partial Iwasawa decomposition

$$
Y=\left(\begin{array}{ll}
V & 0 \\
0 & w
\end{array}\right)\left[\begin{array}{ll}
I & x \\
0 & 1
\end{array}\right]
$$

for $V \in P_{n-1}, w>0, x \in \mathbf{R}^{n-1}$. Then proceed by induction:

It is trivial for $n=1$, so assume it is true for $n-1$. Then

$$
\begin{aligned}
|V| & \leq y_{1} \cdots y_{n-1}, \quad y_{n}=V[x]+w \\
V \in P_{n-1} & \Rightarrow V[x] \geq 0 \Rightarrow w \leq y_{n}, \quad|Y|=|V| w \leq y_{1} \cdots y_{n} .
\end{aligned}
$$

To show $y_{1} \cdots y_{n} \leq\left(\frac{4}{3}\right)^{n(n-1) / 2}|Y|$, again use induction. For $n=$ 2, $|Y|=y_{1} y_{2}-y_{12}^{2}$.

From Lemma 3, $2 y_{12} \leq y_{1}, y_{2}$. Therefore

$$
y_{12}^{2} \leq \frac{1}{4} y_{1} y_{2}
$$

and

$$
|Y| \geq \frac{3}{4} y_{1} y_{2} \quad \text { or } \quad y_{1} y_{2} \leq \frac{4}{3}|Y| .
$$

Assume the inequality for $n-1$. 
If

$$
\begin{gathered}
Y=\left(\begin{array}{cc}
v & 0 \\
0 & W
\end{array}\right)\left[\begin{array}{cc}
1 & T_{X} \\
0 & I
\end{array}\right], \quad W \in F_{n-1} \\
w_{1} \cdots w_{n-1} \leq\left(\frac{4}{3}\right)^{(n-1)(n-2) / 2}|W| \\
y_{1}=v, \quad y_{i}=v x_{i-1}^{2}+w_{i-1} \quad \text { for } i=2, \ldots, n \\
Y \in F_{n} \Rightarrow v \leq v x_{j}^{2}+w_{j} \quad \text { for } j=2, \ldots, n-1 \\
\Rightarrow w_{j} \geq v\left(1-x_{j}\right)^{2} \Rightarrow v \leq \frac{w_{j}}{1-x_{j}^{2}} .
\end{gathered}
$$

Therefore:

$$
y_{i+1} \leq \frac{w_{i}}{1-x_{i}^{2}} x_{i}^{2}+w_{i} \leq w_{i}\left(\frac{x_{i}^{2}}{1-x_{i}^{2}}+1\right) \leq \frac{w_{i}}{1-x_{i}^{2}} \leq \frac{4}{3} w_{i} .
$$

The last inequality is because $\left|x_{i}\right| \leq \frac{1}{2} \Rightarrow x_{i}^{2} \leq \frac{1}{4}$

$$
\begin{aligned}
& \frac{y_{1} \cdots y_{n}}{|Y|}=\frac{y_{1} \cdots y_{n}}{v|W|}=\frac{y_{2} \cdots y_{n}}{|W|} \leq\left(\frac{3}{4}\right)^{n-1} \frac{w_{1} \cdots w_{n-1}}{|W|} \\
& \frac{y_{1} \cdots y_{n}}{|Y|} \leq\left(\frac{3}{4}\right)^{n-1}\left(\frac{3}{4}\right)^{(n-1)(n-2) / 2}=\left(\frac{4}{3}\right)^{n(n-1) / 2} .
\end{aligned}
$$

To prove the last statement of the Theorem, again we appeal to Lemma 3 . We have $y_{1} \leq y_{i}$. Since we have just shown

$$
|Y| \geq\left(\frac{3}{4}\right)^{n(n-1) / 2} y_{1} \cdots y_{n}
$$

the proof follows directly.

COROLlary. Let $Y \in S F_{n}$ where $S F_{n}$ is the fundamental domain for $S P_{n} / \Gamma$. Recall that $S P_{n}=\left\{Y \in P_{n}|| Y \mid=1\right\}$. Then $|W| \geq\left(\frac{3}{4}\right)^{(n-1) / 2}$ where the notation is as in (4.1).

Note that this is consistent with the classical Poincare upper halfplane theory. Recall the correspondence

$$
\begin{gathered}
H \leftrightarrow S P_{n} \\
x+i y=z \rightarrow Y=\left(\begin{array}{cc}
y^{-1} & 0 \\
0 & y
\end{array}\right)\left[\begin{array}{ll}
1 & x \\
0 & 1
\end{array}\right] .
\end{gathered}
$$

For $z \in D, y \geq \sqrt{3} / 2=\left(\frac{3}{4}\right)^{(n-1) / 2}$ when $n=2$. Also, since $v|W|=1$, the statment in the corollary is equivalent to $v \leq\left(\frac{4}{3}\right)^{(n-1) / 2}$.

We now want to consider the notion of cusps of the fundamental domain. In the standard version of the fundamental domain $D$ for the Poincare upper half-plane, there is one cusp in $D$. This is the point at 
infinity, i.e., the point $i y$ where $y \rightarrow \infty$. Due to the inductive nature of the method used to construct $F_{n}$ we might expect that $F_{n}$ should have $n-1$ cusps, which in fact turned out to be the case. However, we have to stretch the generalization of what we mean by a cusp. To illustrate, let us consider the case of $n=3$. We will actually be looking at $S F_{n}$, the fundamental domain for the matrices of determinant 1 in $P_{n}$. If $Y \in S F_{3}, v|W|=|Y|=1$ so $|W|=v^{-1}$. Therefore it makes sense to write

$$
Y=\left(\begin{array}{cc}
v & 0 \\
0 & v^{-1 / 2} W
\end{array}\right)\left[\begin{array}{cc}
1 & T x \\
0 & I
\end{array}\right]
$$

with $|W|=1$. This will mean $W \in S F_{2}$. If

$$
W=\left(\begin{array}{cc}
w & 0 \\
0 & w^{-1}
\end{array}\right)\left[\begin{array}{ll}
1 & u \\
0 & 1
\end{array}\right]
$$

we run into the cusp of $S F_{2}$ as $w \rightarrow 0$. By Lemma 1 ,

$$
\frac{3}{4} v \leq v^{-1 / 2} w \text {, or } \frac{3}{4} v^{3 / 2} \leq w \text {. }
$$

Therefore $w \rightarrow 0 \Rightarrow v \rightarrow 0$. But $v \rightarrow 0$ does not necessarily imply that $w \rightarrow 0$, and yet as $v \rightarrow 0, Y$ approaches the open boundary of $S F_{3}$. This shows that we don't really have a cusp as in a point, but rather $v=0$, a portion of a hyperplane, serves the same purpose. On the other hand, as the cusp of $S F_{2}$ is approached, that is $w \rightarrow 0$, then $v \rightarrow 0$. Thus, $v=0, w=0$ might be called a "second order cusp," since it is a cusp of $S F_{3}$, but also of $S F_{2}$. To generalize, if $Y \in S F_{n+1}$ (we use $n+1$ instead of $n$ to simplify the notation later) we can write

$$
Y=\left(\begin{array}{cc}
v & 0 \\
0 & v^{-1 / n} W
\end{array}\right)\left[\begin{array}{cc}
1 & T X \\
0 & I
\end{array}\right], \quad W \in S F_{n} .
$$

$S F_{n+1}$ will then have $n$ cusps, the primary one as $v \rightarrow 0$, and other, higher order cusps, which can be obtained by reducing $Y$ to its full Iwasawa decompostion by repeating the process above. As mentioned previously, the fundamental domain $F_{n}$ is of interest in obtaining an explicit generalization of the Maass-Selberg relations to $\mathrm{SL}_{3}(Z)$. For it to be useful, however, it must be shown that the fundamental domain has the right "shape" as it goes toward the primary cusp, $v=0$. In other words, it is necessary to prove the following:

THEOREM 3. There exists an $r>0$ such that for $v \leq r$ the set $S$ defined by

$$
\begin{array}{r}
S=\left\{Y \in S P_{n+1} \mid 0<v \leq r, W \in S F_{n} 0 \leq x_{1} \leq \frac{1}{2},\right. \\
\left.\left|x_{j}\right| \leq \frac{1}{2} \text { for } j=2, \ldots, n\right\}
\end{array}
$$

is contained within $S F_{n+1}$ where the notation is as in (5.2). 
Proof. To see this, first let $W^{\prime}=v^{-1 / n} W$, so $W^{\prime} \in F_{n}$. Then recall that if $\lambda$ is the smallest eigenvalue of $W^{\prime}$, then $W^{\prime}[c] \geq \lambda I[c]$ for all $c \in \mathbf{Z}^{n}$. If $r=\lambda$, then for $Y \in S$,

$$
v \leq r \leq \lambda I[c] \leq W^{\prime}[c] \leq v\left[a+{ }^{T} x c\right]+W^{\prime}[c] \text { for } c \neq 0 \text { and all } x \text {. }
$$

Thus, for $Y \in S$, condition (1) in the definition of $F_{n+1}$ is satisfied. Conditions (2) and (3) are clearly satisfied by definition of $S$. Therefore $S \subseteq S F_{n+1}$.

So far, we have discussed some of the boundaries of $F_{n}$. The conditions on the $x$ co-ordinates (condition (3) in the definition) determine $2(n-1)$ boundaries, 2 for each co-ordinate in the vector $x$, all of which are portions of hyperplanes. Condition (2) of the definition of $F_{n}$ tells us that there is a copy of $F_{n-1}$ somewhere in $F_{n}$. As $v \rightarrow 0$, the condition $v \leq v\left[a+T_{x c}\right]+W[c]$ becomes $W[c] \geq 0$. Since this holds for all $W \in F_{n-1}$ (indeed for all $W \in P_{n-1}$ ) we have the copy of the next lower dimension fundamental domain at the cusp $v=0$. If we repeat this process for the fundamental domain $F_{n-1}$ and so on, until we reach $F_{2}$, we obtain a clearer picture of the cusps of $F_{n}$. We see that the fundamental domain $F_{n}$ has cusps that are dependent on all the lower dimensional fundamental domains. These fundamental domains are not compact, but it is always possible to compactify by adding the cusps. Satake obtained a compactification for the fundamental domain for the Siegel modular group in [19], which in many ways appears to be analogous to what would be done for $F_{n}$. If we denote the Siegel fundamental domain of degree $n$ by $S_{n}$, the Satake compactification of $S_{n}$ is obtained by adding the fundamental domains of lower degree. Satake denotes this as $S_{n}^{*}$ and shows that $S_{n}^{*}=S_{n} \cup S_{n-1} \cup S_{n-2} \cup \cdots \cup S_{1} \cup S_{0}$ is a compact closure of $S_{n}$. Here, $S_{1}$ is really the same as $D=H / \mathrm{SL}_{2}(\mathbf{Z})$ and so $S_{0}$ is the usual cusp for $D$, the point at infinity. It should be possible to do the same for $F_{n}$, but this is straying a bit far from the original scope of this paper, and so the details will have to appear later.

6. A more detailed view of the fundamental domain. In this section we will examine more closely condition (1) in the definition of $F_{n}$. Specifically, we will determine explicitly the necessary and sufficient $g \in \Gamma$ that are needed in condition (1) for some small values of $n$. More accurately, since condition (1) depends only on the first column 
of $g$, and it is possible that more than one $g \in \Gamma$ have the same first column, we will determine the necessary and sufficient $a$ and $c$.

As before, for simplicity of notation, let $Y \in F_{n+1}$. Then

$$
Y=\left(\begin{array}{cc}
v & 0 \\
0 & W
\end{array}\right)\left[\begin{array}{cc}
1 & T_{X} \\
0 & I_{n}
\end{array}\right]
$$

where $v>0, W \in F_{n}$, etc. Since $W \in F_{n}$, we can write it as:

$$
W=\left(\begin{array}{cc}
v^{\prime} & 0 \\
0 & W^{\prime}
\end{array}\right)\left[\begin{array}{cc}
1 & T \\
0 & I_{n-1}^{\prime}
\end{array}\right], \quad W^{\prime} \in F_{n-1} .
$$

Then $v\left[a+{ }^{T} x c\right]+W[c]=v\left[a+{ }^{T} x c\right]+v^{\prime}\left[c_{1}+{ }^{T} x^{\prime} c^{\prime}\right]+W^{\prime}\left[c^{\prime}\right]$ where $T_{c}=\left(c_{1}, \ldots, c_{n}\right)$ and ${ }^{T} c^{\prime}=\left(c_{2}, \ldots, c_{n}\right)$. If this process is repeated, we will eventually get to $W^{(n-2)}$ which is an element of $F_{2}$. Let $M=$ $W^{(n-2)}$. Then

$$
v\left[a+T_{X c}\right]+W[c]=v\left[a+T^{T} x\right]+\cdots+M\left[\begin{array}{c}
c_{n-1} \\
c_{n}
\end{array}\right], \quad M \in F_{2} .
$$

Applying Lemma 1 we know that $m_{1} \geq\left(\frac{3}{4}\right)^{n-1} v$ where $M=\left(\begin{array}{cc}m_{1} & m_{12} \\ m_{12} & m_{2}\end{array}\right)$. What can be gathered from this, is that if $M\left[\begin{array}{c}c_{n-1} \\ c_{n}\end{array}\right] \geq\left(\frac{4}{3}\right)^{n-1} m_{1}$, then $v\left[a+{ }^{T} x c\right]+W[c] \geq v$ for all $x$ and $W$, and there is no boundary here. So, we begin by finding the $c_{n-1}$ and $c_{n}$ such that $M\left[\begin{array}{c}c_{n-1} \\ c_{n}\end{array}\right]<\left(\frac{4}{3}\right)^{n-1} m_{1}$ and build up $c$ from there.

As the first example, the boundary conditions for $Y \in F_{3}$ can be obtained using this procedure. $Y=\left(\begin{array}{cc}v & 0 \\ 0 & W\end{array}\right)\left[\begin{array}{ll}1 & T_{x} \\ 0 & I_{2}\end{array}\right]$ where $W \in F_{2}$ and ${ }^{T} x=\left(x_{1}, x_{2}\right)$. Condition (1) says that $v \leq v\left[a+{ }^{T} x c\right]+W[c]$. From the above reasoning it can be seen that the necessary $c$ are those with $W\left[\begin{array}{l}c_{1} \\ c_{2}\end{array}\right]<\frac{4}{3} w_{1}$. Now $W\left[\begin{array}{l}c_{1} \\ c_{2}\end{array}\right]=c_{1}^{2} w_{1}+2 c_{1} c_{2} w_{12}+c_{2}^{2} w_{2}$. We know from Proposition 1 that $F_{2}=M_{2}$ and for $W \in M_{2}, 0 \leq 2 w_{12} \leq w_{1} \leq w_{2}$. Therefore

$$
W\left[\begin{array}{l}
c_{1} \\
c_{2}
\end{array}\right] \geq w_{1}\left(c_{1}^{2}-\left|c_{1} c_{2}\right|+c_{2}^{2}\right)=w_{1}\left(\left(\left|c_{1}\right|-\left|c_{2}\right|\right)^{2}+\left|c_{1} c_{2}\right|\right) .
$$

It can clearly be seen that for $c_{j} \geq 2$, for either $j=1$ or $2, W\left[\begin{array}{l}c_{1} \\ c_{2}\end{array}\right]>$ $2 w_{1}$, so we need only consider $\left|c_{j}\right| \leq 1$. In other words, each $c_{j}$ could be 0 or \pm 1 . This gives nine possible combinations for the vector $c$. As always we discount the possibility $c=0$. This leaves eight nontrivial choices. Recall from the discussion in the previous section that since if $a$ and $c$ form the first column of a $g \in \Gamma$, then so do $-a$ and $-c$, only half of the $c$ need to be looked at further here. Then there were four. These are as follows: $c=\left(\begin{array}{l}1 \\ 0\end{array}\right),\left(\begin{array}{l}0 \\ 1\end{array}\right),\left(\begin{array}{c}-1 \\ 1\end{array}\right),\left(\begin{array}{l}1 \\ 1\end{array}\right)$. Finally $W\left[\begin{array}{l}1 \\ 1\end{array}\right]=w_{1}+2 w_{12}+w_{2} \geq 2 w_{1}>\frac{4}{3} w_{1}$. Thus, the vector $c=\left(\begin{array}{l}1 \\ 1\end{array}\right)$ can 
be thrown out as well. This leaves $c=\left(\begin{array}{l}1 \\ 0\end{array}\right),\left(\begin{array}{l}0 \\ 1\end{array}\right),\left(\begin{array}{c}-1 \\ 1\end{array}\right)$. To determine $a$, consider the inequality $v \leq v\left(a+c_{1} x_{1}+c_{2} x_{2}\right)^{2}+W[c]$ once more. First,

$$
W\left[\begin{array}{l}
1 \\
0
\end{array}\right]=w_{1}, \quad W\left[\begin{array}{l}
0 \\
1
\end{array}\right]=w_{2}, \quad W\left[\begin{array}{c}
-1 \\
1
\end{array}\right]=w_{1}-2 w_{12}+w_{2} .
$$

Since $2 w_{12} \leq w_{2}, W\left[\begin{array}{c}-1 \\ 1\end{array}\right] \geq w_{1}$. Also, $w_{2} \geq w_{1}$. Thus, for the choices of $c$ above, $W[c] \geq w_{1}$. Now, Lemma 1 said that $w_{1} \geq \frac{3}{4} v$. Therefore, for $a$ such that $\left(a+c_{1} x_{1}+c_{2} x_{2}\right)^{2} \geq \frac{1}{4}$, the right hand side of the condition (1) inequality will automatically be greater than $v$. This tells us that we can restrict $a$ so that

$$
\left(a+c_{1} x_{1}+c_{2} x_{2}\right)^{2}<\frac{1}{4} \quad \text { or } \quad\left|a+c_{1} x_{1}+c_{2} x_{2}\right| \leq \frac{1}{2} .
$$

For $c=\left(\begin{array}{l}1 \\ 0\end{array}\right)$ or $\left(\begin{array}{l}0 \\ 1\end{array}\right) a-\frac{1}{2} \leq a+c_{1} x_{1}+c_{2} x_{2} \leq a+\frac{1}{2}$.

If $a \neq 0,\left|a+c_{1} x_{1}+c_{2} x_{2}\right| \geq \frac{1}{2}$ so $a$ must be 0 .

For $c=\left(\begin{array}{c}-1 \\ 1\end{array}\right) a-1 \leq a+c_{1} x_{1}+c_{2} x_{2} \leq a+\frac{1}{2}$ so $a$ could be 0 or 1 .

With the above, condition (1) of the definition of $F_{n}$ can be written explicitly for $n=3$ as follows:

$$
\begin{gathered}
v \leq v\left[a+{ }^{T} x c\right]+W[c] \text { for } a=0 ; \quad c=\left(\begin{array}{l}
1 \\
0
\end{array}\right),\left(\begin{array}{l}
0 \\
1
\end{array}\right) \\
a=0,1 ; \quad c=\left(\begin{array}{c}
-1 \\
1
\end{array}\right)
\end{gathered}
$$

for a total of four inequalities. Conditions (2) and (3) are as before.

If

$$
W=\left(\begin{array}{cc}
w_{1} & 0 \\
0 & w_{2}
\end{array}\right)\left[\begin{array}{cc}
1 & x_{3} \\
0 & 1
\end{array}\right]
$$

this can also be written as $F_{n}$ in the intersection of the following:

(i) $v \leq v x_{1}^{2}+w_{1}$

(ii) $v \leq v x_{2}^{2}+w_{1} w_{3}^{2}+w_{2}$

(iii) $v \leq v\left(x_{1}-x_{2}\right)^{2}+w_{1}\left(1-x_{3}\right)^{2}+w_{2}$

(iv) $v \leq v\left(1-x_{1}+x_{2}\right)^{2}+w_{1}\left(1-x_{3}\right)^{2}+w_{2}$

along with the domains defined by conditions (2) and (3).

An equivalent formulation is if $v^{*}$ is the upper left corner of $Y[g]$, that is $v^{*}$ is the inverse of the height of $Y[g]$, then we must have $v \leq v^{*}$ for

$$
g=\left(\begin{array}{lll}
0 & 1 & 0 \\
1 & 0 & 0 \\
0 & 0 & 1
\end{array}\right), \quad\left(\begin{array}{lll}
0 & 1 & 0 \\
0 & 0 & 1 \\
1 & 0 & 0
\end{array}\right), \quad\left(\begin{array}{rrr}
0 & 1 & 0 \\
1 & 0 & 0 \\
-1 & 0 & 1
\end{array}\right), \quad\left(\begin{array}{rrr}
1 & 0 & 0 \\
-1 & 1 & 0 \\
1 & 0 & 1
\end{array}\right)
$$


Of course, this is just one example of a set of $g$ that completely determine the upper boundary of $F_{3}$. The first columns are the only things that cannot be changed.

Before proceeding on to $n=4,5$ and beyond let us consider a generalization of a statement made above. If $Y \in F_{n+1}$, is it true that $Y[c] \geq y_{1}$ for all $c \in \mathbf{Z}^{n}, c \neq 0$ ? The answer was yes when $n$ was 2, above. For any $n$, if $Y$ has the usual partial Iwasawa decomposition, then

$$
\begin{gathered}
Y[c]=v\left[c_{1}+{ }^{T} x c^{\prime}\right]+W\left[c^{\prime}\right] \text { where }{ }^{T} c=\left(c_{1}, c_{2}, \ldots, c_{n}\right) \\
\text { and }{ }^{T} c^{\prime}=\left(c_{2}, \ldots, c_{n}\right) .
\end{gathered}
$$

By the definition of $F_{n}, v \leq v\left[a+{ }^{T} X c^{\prime}\right]+W\left[c^{\prime}\right]$ for all $a, c^{\prime}$ forming the first column of a matrix in $\Gamma$. From Lemma 2 we see that if g.c.d. $\left({ }^{T} c\right)=1$ then $c$ can be the first column of some $g \in \Gamma$. In any case let $d=$ g.c.d. $\left({ }^{T} c\right)$, and let $c^{*}=d c$. Then $Y[c]=d^{2} Y\left[c^{*}\right]$ and g.c.d. $\left({ }^{T} c^{*}\right)=1$. Therefore $Y[c] \geq Y\left[c^{*}\right] \geq v=y_{1}$. It follows directly from this argument and Lemma 1 that $W[c] \geq \frac{3}{4} v$. We have thus proved the following.

THEOREM 4. If $Y \in F_{n}, Y=\left(\begin{array}{cc}v & 0 \\ 0 & W\end{array}\right)\left[\begin{array}{cc}1 & x \\ 0 & I\end{array}\right]$ then $W[c] \geq \frac{3}{4} v$ for all $c \in \mathbf{Z}^{n-1}, c \neq 0$.

To find the appropriate conditions for $F_{4}$, begin by finding all

$$
c=\left(\begin{array}{l}
c_{1} \\
c_{2} \\
c_{3}
\end{array}\right),
$$

with $c_{2}$ and $c_{3}$ such that $M\left[\begin{array}{l}c_{2} \\ c_{3}\end{array}\right]$ is not automatically greater than $16 / 9 m_{1}$. Since $16 / 9<2$ and $c_{2}, c_{3} \in \mathbf{Z}$ the $c_{2}$ and $c_{3}$ will have the same restrictions as in the case of $F_{3}$, namely $\left|c_{2}\right| \leq 1$ and $\left|c_{3}\right| \leq 1$, but $c_{2}$ and $c_{3}$ are not both 1 or -1 . It can also easily be seen that $\left|c_{1}\right| \leq 1$, since $c_{1}$ here acts like the $a$ from the previous example. This means that there are seven pairs of $c_{2}$ and $c_{3}$ and three possible $c_{1}$ for a total of 21 possible $c$. We discard $c=0$ as usual and consider half the remaining $c$ as before. Of the ten left, it is easily seen that

$$
\left(\begin{array}{l}
1 \\
1 \\
0
\end{array}\right) \text { and }\left(\begin{array}{l}
1 \\
0 \\
1
\end{array}\right)
$$


are unnecessary, as the component of 0 reduces the problem to that of the previous case. This leaves eight choices of $c$ :

$c=e_{1}, e_{2}, e_{3}, \quad\left(\begin{array}{r}1 \\ -1 \\ 0\end{array}\right), \quad\left(\begin{array}{r}1 \\ 0 \\ -1\end{array}\right), \quad\left(\begin{array}{r}0 \\ 1 \\ -1\end{array}\right), \quad\left(\begin{array}{r}1 \\ 1 \\ -1\end{array}\right), \quad\left(\begin{array}{r}1 \\ -1 \\ 1\end{array}\right)$.

Using the Lemma above, it is seen that $|a| \leq 1$ and more explicitly the fundamental domain $F_{4}$ is defined by:

$$
v \leq v\left[a+{ }^{T} x c\right]+W[c]
$$

for

$$
\begin{aligned}
& a=0 ; \quad c=e_{1}, e_{2}, e_{3} \\
& a=0,1 ; \quad c=\left(\begin{array}{r}
-1 \\
1 \\
0
\end{array}\right), \quad\left(\begin{array}{r}
-1 \\
0 \\
1
\end{array}\right) \\
& a=0, \pm ; \quad c=\left(\begin{array}{r}
1 \\
1 \\
-1
\end{array}\right), \quad\left(\begin{array}{r}
1 \\
-1 \\
1
\end{array}\right), \quad\left(\begin{array}{r}
0 \\
-1 \\
1
\end{array}\right)
\end{aligned}
$$

a total of 16 inequalities, along with the inequalities from conditions (2) and (3).

By extending this method $Y \in F_{5}$ the necessary and sufficient $a$ and $c$ are found to be:

$$
\begin{aligned}
& a=0 ; \quad c=e_{1}, e_{2}, e_{3}, e_{4} \\
& a=0,1 ; \quad c=\left(\begin{array}{r}
-1 \\
1 \\
0 \\
0
\end{array}\right), \quad\left(\begin{array}{r}
-1 \\
0 \\
1 \\
0
\end{array}\right), \quad\left(\begin{array}{r}
-1 \\
0 \\
0 \\
1
\end{array}\right) \\
& a=0, \pm 1 ; \quad c=\left(\begin{array}{r}
0 \\
1 \\
-1 \\
0
\end{array}\right), \quad\left(\begin{array}{l}
0 \\
1 \\
0 \\
1
\end{array}\right), \quad\left(\begin{array}{r}
0 \\
0 \\
1 \\
-1
\end{array}\right), \quad\left(\begin{array}{r}
0 \\
1 \\
1 \\
-1
\end{array}\right),\left(\begin{array}{r}
0 \\
1 \\
-1 \\
1
\end{array}\right) \text {, } \\
& \left(\begin{array}{r}
1 \\
1 \\
-1 \\
0
\end{array}\right), \quad\left(\begin{array}{r}
1 \\
1 \\
0 \\
-1
\end{array}\right), \quad\left(\begin{array}{r}
1 \\
0 \\
1 \\
-1
\end{array}\right), \quad\left(\begin{array}{r}
1 \\
-1 \\
1 \\
0
\end{array}\right) \text {, } \\
& \left(\begin{array}{r}
1 \\
-1 \\
0 \\
1
\end{array}\right), \quad\left(\begin{array}{r}
1 \\
0 \\
-1 \\
1
\end{array}\right)
\end{aligned}
$$




$$
\begin{aligned}
a=0, \pm 1, \pm 2 ; \quad c= & \left(\begin{array}{r}
1 \\
1 \\
1 \\
-1
\end{array}\right), \quad\left(\begin{array}{r}
1 \\
1 \\
-1 \\
1
\end{array}\right), \quad\left(\begin{array}{r}
1 \\
1 \\
-1 \\
-1
\end{array}\right), \quad\left(\begin{array}{r}
1 \\
-1 \\
-1 \\
1
\end{array}\right), \\
& \left(\begin{array}{r}
-1 \\
1 \\
-1
\end{array}\right), \quad\left(\begin{array}{r}
1 \\
1 \\
1
\end{array}\right) .
\end{aligned}
$$

This method quickly begins to outlive its usefulness when $Y \in F_{6}$. There are also too many vectors to list, so we will write them in a shorthand form, although this will cost a little accuracy. We first want to find the $c$ where ${ }^{T} c=\left(c_{1}, \ldots, c_{5}\right)$. Unlike the previous case, it is possible for $\left|c_{j}\right|>1$. However, this only happens in a few cases for $c_{4}$ or $c_{5}$. More specifically, $\left|c_{4}\right|,\left|c_{5}\right|=1$ except for the possibilities where one has absolute value 2 while the other has absolute value 1 , and in addition $c_{4} c_{5}<0$. It is also possible for $\left|c_{3}\right|=2$ but that happens only when $\left|c_{4}\right|=1$ and $\left|c_{5}\right|=1$. Finally $\left|c_{2}\right| \leq 2$ and $\left|c_{1}\right| \leq 3$. After determining $c, a$ is found by the same method as before, employing the Lemma of this section. In short, $\left|c_{j}\right| \leq 2$ for $j=2,3,4,5$ and $\left|c_{1}\right| \leq 3$. Then $|a| \leq 4$.

7. The reduction algorithm. In the preceding sections the fundamental domain $F_{n}$ was defined and described. By the way that $F_{n}$ was defined, there is an especially nice algorithm for moving a $Y \in P_{n}$ into $F_{n}$. A point (or matrix) in $F_{n}$ is said to be reduced, and the algorithm is called a reduction algorithm. The reduction algorithm for $F_{n}$ can easily be written as a computer program that will actually perform the operations, as will be seen in the following sections, using $F_{3}$ as an example. For the Minkowski domain, or the similar version of Korkine and Zolotareff, it can be shown that a positive matrix, or equivalently a positive definite quadratic form, can theoretically be reduced, (see [11] for example) but nobody seems to provide an actual method for so doing. This is one more advantage of $F_{n}$.

As might be expected, the algorithm is built up inductively. For $n=2$, the algorithm is just the standard highest point method for the fundamental domain for the upper half-plane. In short, take the starting point, translate until $|x| \leq \frac{1}{2}$, that is act on $Y$ by a matrix of the form $\left(\begin{array}{ll}1 & 1 \\ 0 & 1\end{array}\right)^{k}, k \in \mathbf{Z}$, then flip if necessary, by using the action of the matrix $\left(\begin{array}{rr}0 & 1 \\ -1 & 0\end{array}\right)$. Repeat the process until the point is in the fundamental domain. It will be seen shortly why this process must terminate, that is, why repeating this process will eventually yield a 
point in the fundamental domain. For more details on the $n=2$ case, see [17] among many others.

For the general case, assume the algorithm exists for all $F_{j}$ with $j<n$. Write $Y=\left(\begin{array}{cc}v & 0 \\ 0 & W\end{array}\right)\left[\begin{array}{ll}1 & T_{x} \\ 0 & I\end{array}\right]$ as usual. Then the reduction algorithm consists of the following steps:

Step 1. Put $W \in F_{n-1}$. The induction hypothesis says that this is possible, i.e., there is a matrix $D \in \mathrm{GL}_{n-1}(\mathbf{Z})$ such that $W[D] \in F_{n-1}$, and there is an algorithm for determining $D$. Then act on $Y$ by $g=$ $\left(\begin{array}{ll}1 & 0 \\ 0 & D\end{array}\right)$. Then

$$
Y[g]=\left(\begin{array}{cc}
v & 0 \\
0 & W[D]
\end{array}\right)\left[\begin{array}{cc}
1 & T_{X D} \\
0 & I
\end{array}\right]
$$

Let $Y^{\prime}=Y[g]$ and ${ }^{T} X^{\prime}={ }^{T} X D$.

Step 2. Use translation-type matrices to make the $x$ co-ordinates satisfy condition (1) of the Definition, that is $0 \leq x_{1} \leq \frac{1}{2} ;\left|x_{j}\right| \leq \frac{1}{2}$ for $j=2, \ldots, n-1$. These translation-type matrices are of the form $T_{j}$ where $T_{j}$ has diagonal entries 1 and a 1 in the first row, $j+1$ st column, all other entries being 0 . Write ${ }^{T} x^{\prime}=\left(x_{1}^{\prime}, \ldots, x_{n-1}^{\prime}\right)$ and let $r_{j}=\left[\frac{1}{2}-x_{j}\right]$ where $[\alpha]$ denotes the greatest integer in $\alpha$. Then let

$$
g^{\prime}=\prod_{j=1}^{n-1} T_{j}^{r_{j}} \quad \text { if } 0 \leq x_{1}+r_{1} \leq \frac{1}{2}
$$

and let

$$
g^{\prime}=\left(\prod_{j=1}^{n-1} T_{j}^{r_{j}}\right) U, \quad U=\left(\begin{array}{rr}
-1 & 0 \\
0 & I
\end{array}\right) \quad \text { if }-\frac{1}{2} \leq x_{1}+r_{1}<0 .
$$

In either case the matrix $Y^{\prime}\left[g^{\prime}\right]$ satisfies conditions (2) and (3) of the Definition. This can be seen by observing that

$$
Y^{\prime}\left[g^{\prime}\right]=\left(\begin{array}{cc}
v & 0 \\
0 & W[D]
\end{array}\right)\left[\begin{array}{cc}
1 & T X^{*} \\
0 & I
\end{array}\right]
$$

where $x^{*}= \pm\left(x^{\prime}+r\right)$ with $r$ being the vector with components $r_{j}$ and the sign being chosen as to make $x_{1}+r_{1}$ non-negative. Note that the order in which the product is taken is unimportant, as all the $T_{j}$ commute, but the matrix $U$ must be multiplied on the right if it occurs.

Step 3. If $Y$ is the matrix obtained after Step 2,

$$
Y=\left(\begin{array}{cc}
v & 0 \\
0 & W
\end{array}\right)\left[\begin{array}{cc}
1 & T_{X} \\
0 & I
\end{array}\right]
$$

then $W \in F_{n-1}$ and $0 \leq x_{1} \leq \frac{1}{2}$ and $\left|x_{j}\right| \leq \frac{1}{2}$ for $j=2, \ldots, n-1$. Consider the set of the $v\left[a+{ }^{T} x c\right]+W[c]$ for all the $a$ and $c$ defining the 
necessary and sufficient inequalities in condition (1) of the Definition. Choose $v^{*}$ to be the minimal element of that set, and let $g^{*}$ be an element of $\mathrm{GL}_{n}(\mathbf{Z})$ with the first column determined by the $a$ and $c$ from $v^{*}=v\left[a+{ }^{T} x c\right]+W[c]$. Replace $Y$ by $Y\left[g^{*}\right]$.

Repeat Steps 1, 2, and 3 until $Y \in F_{n}$ is obtained. To see that this process must eventually terminate, recall from the proof of Theorem 1 , that if $\lambda$ is the smallest eigenvalue of $Y$, then $Y[c] \geq \lambda I[c]$ $=\lambda\left(c_{1}^{2}+\cdots+c_{n-1}^{2}\right)$, and this shows that there can be only finitely many $a$ and $c$ such that $v\left[a+T_{x c}\right]+W[c] \leq m$ for some positive real number $m$. If $m$ is chosen to be the $v$ from the original $Y$, then Step 3 chooses a $v^{*}$ from this finite set with $v^{*} \leq v$. If $v^{*}=v$, then $v \leq v\left[a+{ }^{T} x c\right]+W[c]$ for all the $a$ and $c$ considered, and so, by the Definition, the matrix obtained from Step 3 is in $F_{n}$. Otherwise, $v^{*}<v$, and so the same argument holds with $m=v^{*}$ and a smaller set. Thus it is seen that the algorithm terminates.

Those readers with an affinity for computers will see that this algorithm easily lends itself to being programmed. In performing the algorithm for $n$, one just appeals recursively to the algorithms for the lower dimensional fundamental domains. This is seen more explicitly for $n=3$ in the following section.

8. A reduction algorithm for $P_{3}$. It has been shown $(\S 6)$ that there are four necessary and sufficient $g$ for condition (1) of the Definition in the case $n=3$. Let these be written

$$
\begin{aligned}
& S_{1}=\left(\begin{array}{lll}
0 & 1 & 0 \\
1 & 0 & 0 \\
0 & 0 & 1
\end{array}\right), S_{2}=\left(\begin{array}{lll}
0 & 1 & 0 \\
0 & 0 & 1 \\
1 & 0 & 0
\end{array}\right), \\
& S_{3}=\left(\begin{array}{rrr}
0 & 1 & 0 \\
-1 & 0 & 1 \\
1 & 0 & 0
\end{array}\right), \quad S_{4}=\left(\begin{array}{rrr}
1 & 0 & 0 \\
-1 & 1 & 0 \\
1 & 0 & 1
\end{array}\right)
\end{aligned}
$$

and let

$$
T_{1}=\left(\begin{array}{lll}
1 & 1 & 0 \\
0 & 1 & 0 \\
0 & 0 & 1
\end{array}\right), \quad T_{2}=\left(\begin{array}{lll}
1 & 0 & 1 \\
0 & 1 & 0 \\
0 & 0 & 1
\end{array}\right), \quad \text { and } \quad U=\left(\begin{array}{rrr}
-1 & 0 & 0 \\
0 & 1 & 0 \\
0 & 0 & 1
\end{array}\right)
$$

as defined in Step 2 of the algorithm in the preceding section. Let

$$
M_{1}=\left(\begin{array}{lll}
1 & 0 & 0 \\
0 & 1 & 1 \\
0 & 0 & 1
\end{array}\right), \quad M_{2}=\left(\begin{array}{rrr}
1 & 0 & 0 \\
0 & 0 & 1 \\
0 & -1 & 0
\end{array}\right), \quad M_{3}=\left(\begin{array}{rrr}
1 & 0 & 0 \\
0 & -1 & 0 \\
0 & 0 & 1
\end{array}\right) \text {. }
$$

These last three are of the form $\left(\begin{array}{ll}1 & 0 \\ 0 & D\end{array}\right), D \in \mathrm{GL}_{2}(\mathbf{Z})$, and are used in Step 1 of the algorithm. 
The algorithm for placing $Y \in P_{3}$ then can be given explicitly. Let $Y$ be written in the usual partial Iwasawa form:

$$
Y=\left(\begin{array}{cc}
v & 0 \\
0 & W
\end{array}\right)\left[\begin{array}{cc}
1 & T x \\
0 & I
\end{array}\right] \quad \text { with } W \in P_{2} \quad \text { and } \quad T^{T} x=\left(x_{1}, x_{2}\right) .
$$

Step 1. Since $W \in P_{2}$ it can be written as

$$
W=\left(\begin{array}{cc}
w_{1} & 0 \\
0 & w_{2}
\end{array}\right)\left[\begin{array}{cc}
1 & x_{3} \\
0 & 1
\end{array}\right] .
$$

Let $r_{3}=\left[\frac{1}{2}-x_{3}\right]$ and $M=M_{1}^{r_{3}}$. Replace $W$ by $W[M]$ if $x_{3}+r_{3} \geq 0$ and by $W\left[M M_{3}\right]$ if $x_{3}+r_{3}<0$. Then if $w_{1}>w_{1} x_{3}^{2}+w_{2}$ replace $W$ by $W\left[M_{2}\right]$. Repeat until $0 \leq x_{3} \leq \frac{1}{2}$ and $w_{1} \leq w_{1} x_{3}^{2}+w_{2}$. Then $Y$ satisfies condition (2) of the Definition.

Step 2. Let $r_{j}=\left[\frac{1}{2}-x_{j}\right], j=1,2$. Let $T=T_{1}^{r_{1}} T_{2}^{r_{2}}$. Replace $Y$ by $Y[T]$ if $x_{1}+r_{1} \geq 0$, by $Y[T U]$ if $x_{1}+r_{1}<0$. Then the new $Y$ satisfies conditions (2) and (3).

Step 3. Let $S_{0}=I$, and let $v_{j}$ be the upper left corner of the positive matrix $Y\left[S_{j}\right]$ for $j=0,1,2,3,4$. Note that $v_{0}=v$. Suppose that $v_{k} \leq v_{j}$ for all $j$. Then replace $Y$ by $Y\left[S_{k}\right]$.

Now return to Step 1 and repeat until $Y \in F_{3}$.

9. Applications. The reduction algorithm of the preceding sections is the first example of an application of the new fundamental domain. This in itself shows some of the value of these fundamental domains since, as was mentioned earlier, there do not seem to be any satisfactory means for reducing an element of $P_{n}$ to any of the other types of fundamental domains. There are also applications that make use of this reduction algorithm. In [2], the reduction algorithm for $n=3$ was used to obtain information on Hecke operators for $\mathrm{GL}_{3}(Z)$ and on the density of the images in the fundamental domain of Hecke points, that is, the points used to define the Hecke operators. It may also be possible to use this reduction algorithm to investigate Fourier coefficients for automorphic forms for $\mathrm{GL}_{3}(\mathbf{Z})$ as Stark does for $\mathrm{SL}_{2}(\mathbf{Z})$ in [16]. Another possible application of the reduction algorithm is mentioned in [17, Vol. II]. There it says that a problem of interest is the search for a generalization of the theorem that a number is quadratic if and only if its continued fraction is periodic. The reduction algorithm comes in because the continued fraction of $\alpha$ can be determined by finding the element of $\mathrm{SL}_{2}(Z)$ that maps the semi-circle joining $\alpha$ and its (algebraic) conjugate. A relation between units in real quadratic fields and hyperbolic elements of $\mathrm{SL}_{2}(\mathbf{Z})$ also drops out of this. Minkowski, [9], 
obtained some partial results along the lines of generalizing these ideas to $n>2$, so perhaps with a new fundamental domain further work of this type can be accomplished. Finally, the reduction algorithm for positive quadratic forms has applications to various problems in lattice theory.

Another possibility is to slightly alter the definition of the fundamental domain for the Siegel upper half-plane. Recall that one condition in that definition was $Y \in M_{n}$, where $M_{n}$ was Minkowski's fundamental domain for $P_{n}$. However, the definition only depended on $Y$ being in a fundamental domain for $P_{n}$, and so it is possible to replace the condtion $Y \in M_{n}$ with $Y \in F_{n}$ without changing any of the proof that the domain as defined in $\S 1$ is a fundamental domain for the Siegel upper half-plane. The advantages of tampering with the definition in this way would be that both the fundamental domain for the Siegel upper half-plane and the fundamental domain $F_{n}$ for $P_{n}$ were obtained by generalizing the highest point method and so might be more compatible. For example, it might be possible to further the results of Gottschling in [3] by taking advantage of the inductive nature of $F_{n}$. There are numerous other possibilities in this area since the $F_{n}$ and the fundamental domain for the Siegel upper half-plane are so intimately connected.

Some of the most interesting applications of $F_{n}$ are in the study of automorphic forms on $\mathrm{GL}_{n}(\mathbf{Z})$. Since one of the original factors for developing the fundamental domain $F_{n}$ was to make the task of generalizing the Maass-Selberg relations easier, it is not surprising that this is perhaps the most obvious application aside from the reduction algorithm. Some partial results along these lines, especially for $n=3$, but even for any $n$, have been obtained in [4]. There it is seen that the definition of $F_{n}$ indeed allows one to make use of the Fourier expansion in the $x$ co-ordinates of automorphic forms on $\mathrm{GL}_{n}(\mathbf{Z})$.

\section{REFERENCES}

[1] D. Bump, Automorphic Forms on $\mathrm{GL}(3, R)$, Lecture Notes in Mathematics 1083, Springer-Verlag, New York, 1984.

[2] D. Gordon, D. Grenier, and A. Terras, Hecke operators and the fundmental domain for SL(3, Z), Math. Comp., 48 (1987), 159-178.

[3] E. Gottschling, Explizite Bestimmung der Randflaechen des Fundamentalbereiches der Modulgruppe zweiten Grades, Math. Ann., 138 (1959), 103-124.

[4] D. Grenier, Fundamental Domains for $P_{n} / \mathrm{GL}_{n}(Z)$ and Applications in Number Theory, Ph.D. Thesis, UCSD, 1986.

[5] E. Hecke, Mathematische Werke, Vandenhoeck und Ruprecht, Gottingen, 1970. 

York, 1981.

[7] H. Maass, Siegel's Modular Forms and Dirichlet Series, Lecture Notes in Mathematics 216, Springer-Verlag, New York, 1971.

[8] U Uber eine neue Art von nichtanalytischen automorphen Funktionen und die Bestimmung Dirichletscher Reihen durch Funktional gleichung, Math. Ann., 121 (1949), 141-183.

[9] H. Minkowski, Gesammelte Abhandlungen, Chelsea, New York, 1967.

[10] S. Ryskov, The theory of Hermite-Minkowski reduction of positive definite quadratic forms, J. Soviet Math., 6 (1976), 651-676.

[11] S. Ryskov and E. Baranovskii, Classical methods of the theory of lattice packings, Russian Math. Surveys, 34 (1979), 1-68.

[12] A. Selberg, Harmonic analysis and discontinuous groups in weakly symmetric Riemannian spaces with applications to Dirichlet series, J. Indian Math. Soc., 20 (1956), 47-87.

[13] C. L. Siegel, Gesammelte Abhandlungen, Springer-Verlag, New York, 1979.

[14] , Lectures on Quadratic Forms, Tata Institute of Fundamental Research, Bombay, 1963.

[15] _ Symplectic Geometry, Academic Press, New York, 1964.

[16] H. Stark, Fourier coefficients of Maass wave forms, in Modular Forms, R. A. Rankin (Ed.), Horwood, Chichester, 1984, pp. 263-269.

[17] A. Terras, Harmonic Analysis on Symmetric Spaces and Applications, Vols. I and II, Springer-Verlag, New York, 1985.

[18] A Terras, Some simple aspects of the theory of automorphic forms for GL(n.Z), in The Selberg Trace Formula and Related Topics, D. Hejhal, P. Sarnak, and A. Terras (Eds.), AMS, Providence, 1986.

[19] I Satake, On the compactification of the Siegel space, J. Indian Math. Soc., 20 (1956), 259-281.

Received October 10, 1986 and in revised form February 18, 1987.

The University of TeXas at Austin

Austin, TX 78712 



\title{
PACIFIC JOURNAL OF MATHEMATICS EDITORS
}

\author{
V. S. VARADARAJAN \\ (Managing Editor) \\ University of California \\ Los Angeles, CA 90024 \\ HERBERT Clemens \\ University of Utah \\ Salt Lake City, UT 84112 \\ R. FINN \\ Stanford University \\ Stanford, CA 94305
}

\author{
HERMANN FLASCHKA \\ University of Arizona \\ Tucson, AZ 85721
}

Ramesh A. Gangolli University of Washington Seattle, WA 98195

VAUGHAN F. R. JONES University of California Berkeley, CA 94720
ROBION KIRBY

University of California

Berkeley, CA 94720

C. C. MOORE

University of California

Berkeley, CA 94720

HAROLD STARK

University of California, San Diego La Jolla, CA 92093

\section{ASSOCIATE EDITORS}

\author{
R. ARENS \\ E. F. BECKENBACH \\ B. H. NEUMANN \\ F. WOLF \\ K. YOSHIDA \\ (1906-1982)

\section{SUPPORTING INSTITUTIONS}

\section{UNIVERSITY OF ARIZONA} \\ UNIVERSITY OF BRITISH COLUMBIA \\ CALIFORNIA INSTITUTE OF TECHNOLOGY \\ UNIVERSITY OF CALIFORNIA \\ MONTANA STATE UNIVERSITY \\ UNIVERSITY OF NEVADA, RENO \\ NEW MEXICO STATE UNIVERSITY \\ OREGON STATE UNIVERSITY \\ UNIVERSITY OF OREGON \\ UNIVERSITY OF SOUTHERN CALIFORNIA \\ STANFORD UNIVERSITY \\ UNIVERSITY OF HAWAII \\ UNIVERSITY OF TOKYO \\ UNIVERSITY OF UTAH \\ WASHINGTON STATE UNIVERSITY \\ UNIVERSITY OF WASHINGTON
}

The Supporting Institutions listed above contribute to the cost of publication of this Journal, but they are not owners or publishers and have no responsibility for its content or policies.

Mathematical papers intended for publication in the Pacific Journal of Mathematics should be in typed form or offset-reproduced (not dittoed), double spaced with large margins. Please do not use built up fractions in the text of the manuscript. However, you may use them in the displayed equations. Underline Greek letters in red, German in green, and script in blue. The first paragraph must be capable of being used separately as a synopsis of the entire paper. In particular it should contain no bibliographic references. Please propose a heading for the odd numbered pages of less than 35 characters. Manuscripts, in triplicate, may be sent to any one of the editors. Please classify according to the scheme of Math. Reviews, Index to Vol. 39. Supply name and address of author to whom proofs should be sent. All other communications should be addressed to the managing editor, or Elaine Barth, University of California, Los Angeles, California 90024

There are page-charges associated with articles appearing in the Pacific Journal of Mathematics. These charges are expected to be paid by the author's University, Government Agency or Company. If the author or authors do not have access to such Institutional support these charges are waived. Single authors will receive 50 free reprints; joint authors will receive a total of 100 free reprints. Additional copies may be obtained at cost in multiples of 50 .

The Pacific Journal of Mathematics is issued monthly as of January 1966. Regular subscription rate: $\$ 190.00$ a year (5 Vols., 10 issues). Special rate: $\$ 95.00$ a year to individual members of supporting institutions.

Subscriptions, orders for numbers issued in the last three calendar years, and changes of address should be sent to Pacific Journal of Mathematics, P.O. Box 969, Carmel Valley, CA 93924, U.S.A. Old back numbers obtainable from Kraus Periodicals Co., Route 100, Millwood, NY 10546.

The Pacific Journal of Mathematics at P.O. Box 969, Carmel Valley, CA 93924 (ISSN 0030-8730) publishes 5 volumes per year. Application to mail at Second-class postage rates is pending at Carmel Valley, California, and additional mailing offices. Postmaster: send address changes to Pacific Journal of Mathematics, P.O. Box 969, Carmel Valley, CA 93924.

PUBLISHED BY PACIFIC JOURNAL OF MATHEMATICS, A NON-PROFIT CORPORATION Copyright (C) 1988 by Pacific Journal of Mathematics 


\section{Pacific Journal of Mathematics}

\section{Vol. 132, No. $2 \quad$ February, 1988}

Jeffery Marc Bergen and Luisa Carini, A note on derivations with power central values on a Lie ideal ..............................209

Alfonso Castro and Sumalee Unsurangsie, A semilinear wave equation

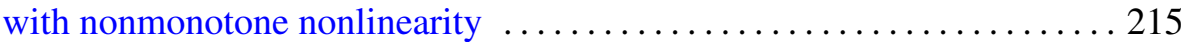

Marius Dadarlat, On homomorphisms of matrix algebras of continuous

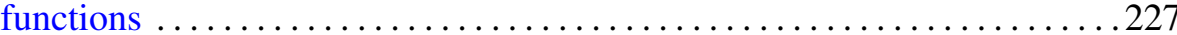

A. Didierjean, Quelques classes de cobordisme non orienté refusant de se

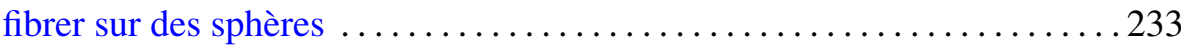

Edward George Effros and Zhong-Jin Ruan, On matricially normed spaces

Sherif El-Helaly and Taqdir Husain, Orthogonal bases are Schauder bases

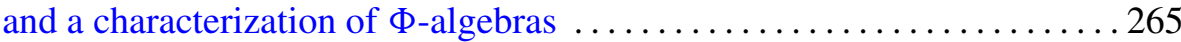

Edward Richard Fadell and Peter N-S Wong, On deforming $G$-maps to be

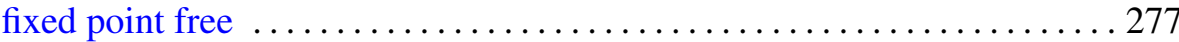

Jean-Jacques Gervais, Stability of unfoldings in the context of equivariant

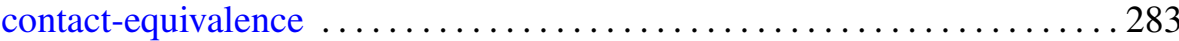

Douglas Martin Grenier, Fundamental domains for the general linear group

Ronald Scott Irving and Brad Shelton, Loewy series and simple projective

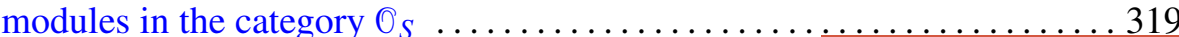

Russell Allan Johnson, On the Sato-Segal-Wilson solutions of the K-dV equation

Thomas Alan Keagy and William F. Ford, Acceleration by subsequence transformations

Min Ho Lee, Mixed cusp forms and holomorphic forms on elliptic varieties

Charles Livingston, Indecomposable surfaces in 4-space 